\title{
"What Could Be the Concept of Time in Relation to Behavior in the Virtual World?"
}

\author{
Nadine Touzeau* \\ Researcher in Cybercriminal Behavior, France \\ *Corresponding author: Nadine Touzeau, Net-profiler, Researcher in Cybercriminal Behavior, France
}

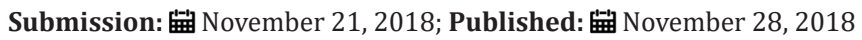

\begin{abstract}
By questioning several people, when asked to define time, they all have a different approach. Their notion of time is specific to their vision, their interpretation of the speed of events in response to their needs and emotions. Many scientists have their own theory of time, which sometimes contradict others. If Galileo [1] has quantified time by mathematical measures, time measurement implicitly presupposes an orientation of time from the past to the future. The speed of the element's movement in space can be constant or not. A measure that allows us to know or even predict the time it will take to do this or that act. Added to the physical aspect, without obscuring Albert Einstein's theory of Relativity [2], the perception of the human being deserves to be integrated. Depending on the profile, emotions, cognition, memory, time duration can be subjective [3] or objective. If everyone agrees that time is irreversible, how can time in a virtual space be measured scientifically from a behavioural point of view? With regard to my scientific theories, "Avatarization", "Virtual Intelligences", "Transverse Zone” developed in my scientific publications [4] and my books concerning Net profiling [5-11] and related my research about behavioural differentiations between the real and the virtual, I wonder about the concept of time in virtual space, this impalpable, fast and interactive space. Certainly, the focus will be on the behaviour of virtual space users, including cybercriminals. Heraclitus [12] and the atomists [13] proposed to confuse matter with movement. According to them, everything is mobile. Since this is the case, how does the human behind the screen integrate and quantify this notion of time?
\end{abstract}

Keywords: Net-profiling; Concept of time; Transverse zone; Cyber space; Virtual space; Behavioral differentiation; Avatarization; Virtual intelligence

\section{Introduction}

When asked about the question of time, Saint Augustine [14] said: "If no one asks me, I know it, but if I am asked and I want to explain it, then I no longer know it. It is from this reflection that I asked myself the question of what state the notion of time in virtual space should be in. Considering that the people using this virtual space are human beings, you and I, cybercriminals, neophytes, scholars, therefore, human beings living in reality, it seems important to me to understand the notion of time in reality before explaining it, if at all possible, in the cyber space.

Time is calculated by different tools: sundial, calendar, watch, etc. All these means make it possible to measure time space in our universe, our own space. A measurement based on many scientists' works from various backgrounds that allows us to frame space time in our daily lives. However, many people do not have the same relationship to time as others? Some find it long, others short, depending on the extent of the context or the moment he lives it. However, space time is the same and fixed according to the scientific criteria chosen for a universal measurement. Waiting, feeling, envy, hope, stress, fear, are just some of the emotions that alter the notion of time and make it unique to everyone.

This is how time can be measured according to affective, emotions, experiences, preconceptions, objectives, or according to purely personal perceptions. Time can be suspended following a strong event from which we have an urgent expectation. The time estimate can be perceived as very long while the space time was short. A minute becomes an hour, and conversely on events that make you forget time. These same human beings use virtual space. A space where the notion of mathematical, physical time should, in my opinion, integrate the human being into their calculation and analysis. The reason is simple: men use this space according to their perception of time, as they experience it in the real world, with a space-time measurement that has been inked for years in one universe or space-time in another dimension.

A fast dimension that can be measured by the click or by the fact that the space is transversal, without borders or limits and accessible everywhere in the world by not moving any space. This confrontation of man in this space where time no longer has the same understanding of behavioural and scientific patterns challenges me. Morvan Salez [15] gives his own definition of time: "Time is extremely subjective. We are culturally trapped by the idea that we live physical time and perceive it with our consciousness, but I think that in fact, the time that our consciousness perceives is something that resembles physical time, has its flavor, seems to take the same direction, to go at the same pace but that it is not physical time. This notion of linear time is an illusion that you make 
yourself and that resembles physical time. "And if this vision could adapt to virtual space".

\section{Discussion}

Etienne Klein [16] puts forward another vision of time. He thinks that the main difficulty is related to language, which tends to confuse time and temporal phenomena. The word "time" is used to mean something other than itself. It is used to refer to aging, succession, simultaneity, duration, etc.

Based on this thought, considering that there is an end, for example, can truncate the notion of time, especially if the end is known. This overlaps with the fact that the notion of time is specific to each individual despite being represented with a mathematics and physical measure. The minute that corresponds to 60 seconds may seem short or long, or even have no impact on a human being. Who hasn't found the time to spend in an anxious situation, a context of stress, an endless wait? And who has not estimated the short time by wondering about the late hour after a beautiful evening, a day too short to have experienced a moment of relaxation, years of happiness with the loved one you do not see growing old?

The space-time that runs because of its scientific representation has no control over people. It is unique to each of us. Look at his watch every minute indicates in behavioral terms the annoyance and expectation, that is, that the time is not in phase with the individual's need. Just as forgetting to look at your watch shows a lack of need to know the time, a free time or the desire to live the moment that time could stop. The measurement of the time of phenomena in science is finally little integrated into the behaviour of the human being who appropriates himself according to his profile, emotions, objectives, capacities, potentials, desires, etc. Events also alter the notion of time. Lost memory that promotes forgetting and makes you lose track of time. The past forces us to reflect in order to know when the facts took place, to verify the accuracy of the memory, to put it in context.

The disease can temporarily alter the perception and awareness of time quite often. A trauma suffered after a shock is an example. Events of which we are sometimes unaware modify our perception of time in temporal space. We experience this time at the rhythm of these events whether they are joyful, programmed, desired, accidental, controlled, impromptu. With the certainty that they can never go back, stop or manipulate it. We experience time to the rhythm of the hands of a watch by perceiving it according to the context and the moment, the experience and emotions, the desire and the spirit.

Some cultures consider that we should not rush and that time has no control over them. They live according to what must be done without imposing what is not wanted at the moment. Like a mission to be accomplished whose path is to be followed without discussion. Time is only an evidence and not an annoying or obstructive notion. The point is that things must be done as they are written. Like a destiny where time has neither taken nor taken its place. Just to accomplish his mission. These people are often very calm and develop a certain wisdom of life that is not stressful. They do what must be done to achieve their life goal.

Others are thirsty to live as much as possible in a short time, based on the fact that life must be fully lived by what it passes quickly. A willingness that can generate stress. Time somehow directs everyday life. This time according to what they interpret it. The fact of living what they desire animates their daily lives, even if it means neglecting the quality of the event and the values of life. Do everything and quickly before you die or it's too late. Live like everyone else to feel fashionable or be the first. It is necessary to live things quickly without time altering actions.

It should be noted that the haste to live quickly in the moment can make the short time seem so obvious. For example, every week- end we will experience a different cruise, waiting for the moment to make time long, and, once on a cruise, time will not be felt out of happiness to live the moment for example. This allows me to bounce back on the users of virtual space. Some profiles also tend to want everything right away. Online shopping meets high expectations and the one-click shopping experience satisfies the customer. I want, I click and I have. The notion of time is extremely fast, especially since the person does not have to travel, organize his time to carry out the process, use public transport, look for his parking, risk not finding the object in the store. As described in one of my theories "Transverse Zone"[4], behaviour changes according to the event that occurs on its connected object or its relationship to the virtual. Time in the virtual world is very much linked to the "click" on the Internet.

The fact of having everything quickly from your chair all over the world, having access in a few links on an internet search engine to the expected source, buying without stock limits and the requirement to litter shopping streets and respect shop hours, talking with a virtual person anywhere in the world, in short doing almost anything you want behind your screen in a short time with a completion of the act that is called a click, favours changing your approach over time.

I will take the example of the e-mail when it invaded our professional environment. Sever- al people have transposed the email as a facilitator into communication. Accessibility came from the user to the recipient to expect from the recipient an equally comfortable response in the quickest way possible. That is to say, they considered that the mail stole the verbal communication used before it invaded our lives. Thus, emails were constantly written, for a lesser pretext, because they were easily accessible in order to transmit information in a very short period of time. Sometimes the email had no form of politeness and was ad- dressed to the person right in front of his office.

The recipient of the email was sometimes in front of the office. In a single day, he could receive several from the same person. While before both of them were talking over coffee, they moved around to get the elements and in-formation that the email missions. The con- tact, the non-verbal, the physical movement, the resulting 
exchanges, the explanations concerning the subject requested, the human warmth that fueled communication often starting with a hello, a hand wrist, a kiss, dis- appeared in the email.

Without moving, communication is done by machine, without exchange, without sharing, without human contact. A communication that has been acquired over time and transposed into other exchanges ranging from post to chat. In reality, it is a new form of communication that makes humans disappear and al-lows contact everywhere on the virtual planet for the most part. At the time of the beginning of email, this created frustrations since the mail transposed the communication while the content of an email is often brief and without exchange.

Time. Save time without moving from your desk by sending requests and information by email to whoever you want without limit of border, time, space, language, subject. It only takes a few seconds, or minutes, without moving from your workstation, to do what you used to do in minutes or hours, or even couldn't do, by email. All over the world. Physical travel is no longer necessary, making appointments or waiting for the phone to pick up is no longer necessary. Just as you read your messages whenever you want. We answer them if we want. The email has changed the notion of time between reality and the virtual in humans.

Communication between colleagues before the virtual was often limited to office hours. Email has no time, no days, no time to be written from wherever you want, from your smartphone, on the beach making it look like you're working in an office, on a Saturday night on earth! This example has had an impact on all acts performed in the virtual world, changing the behaviour of users according to profiles and targets of the users. Used to the speed of sending an email and getting a response by return quickly, impatience can arise faster in some individuals once the email response will take time to come. This is also true of chat. The interaction of written exchanges on an instant messenger in almost as fast a time as if the person was speaking in front of you can put a strain on patience if the interaction of the exchanges is slow. The pace of written exchanges must be now and instantaneous. Moreover, the language has been modified because it has been simplified and represented by images expressing emotions or desires.

Waiting for a response or even reading the message can cause stress, need, questions. Re- activity in the virtual world has created its behaviours whereas in the past, we waited to see or hear the person for interactivity. We respected the response time of a letter sent by post while not getting a reply by return mail can become unbearable. However, users of virtual space live in reality! This impatience can also be seen in the real world. Here again, used to the comfort of speed, a too long queue can annoy more quickly than not. Not having the product in stock in a shop and having to return as soon as it is available can cause the sale to fail.

Waiting becomes more difficult in real life due to the ease of time management in the virtual world, the achievement of objectives, and challenges the work of patience. The generation born with the Internet may not even be interested in working on patience. It would be interesting to know their definition. The press article [17] cites that Generation Y, the first generation considered to have been born with the Internet, is: "Everything we want has become instant access." This suggests that patience may be absent, misunderstood or even unknown. In any case, it is put to the test.

If we also analyses exchanges of dredges, in the French language familiarization is only considered if we know each other and agree to use it. This familiarization is very quickly used from the very first exchanges that can make the recipient of the message uncomfortable. It can result in a request for an appointment just as quickly and a sexual relationship even before meeting. This leads to misunderstandings and discrepancies between cultures, education and generations. An educated woman will not accept such an approach and may be perceived as outdated when she cannot give emotional support virtually.

Even at this level, consumption is fast. Moreover, in reality, this can be felt in men's more eager behaviour towards women, as they dare to do in the virtual world. Knowing that reality confronts the person physically. Few pro- files dare in reality what the virtual offers in ease. The time of emotions can also be changed due to the virtual and also to have virtual flirting and sexual exchanges with strangers in one click.

The intimacy goes from "Hello, I like you" to "You excite me, I send you the picture of my erect sex" in a few steps of the chat to discover the person and his privacy. The notion of time seems to have no effect on the discovery of the other to take it for a consumable like buying a sweater on an online clothing site. Time in the virtual no longer seems to correspond to the notions we had in real space. Reality already had difficulty agreeing on this notion of time and how to measure it in terms of patience, desires, expectations, stress, etc. The virtual, which favours speed, modifies this notion of time both in the virtual and in the real world.

Does this promote "Avatarization" [4], these behaviors created in and be- cause of virtual space sometimes duplicated in reality. I think so, since these behaviors are born from the ease and speed of creating them behind the screen. A facility that does not encounter a prohibition brake, so a time not to be managed to avoid, repair or simply plan that these prohibitions do not hinder the implementation of the Avatar. As for "Virtual Intelligence" [4], users of virtual space have developed potential for speed and adaptability in an environment different from their own, the one in which they live. This speed and ability to "live" in this cyber space also alters the notion of time defined by the many scientists experienced in reality.

Cybercriminals and frequent users of virtual space are familiar with the fast functioning of the virtual. They use it in the expectations of each of their actions in this space that must not and cannot exceed what they know about it, often around a simple click. The virtual has accelerated the ability to quickly obtain what we want by eliminating the steps that reality needs to experience. 
However, transposing the notion of time lived in the virtual, in the real world, could also al- low these steps to disappear. Patience, waiting, reflection, discovery are all assets that can disappear from the individual in order to adapt to a new universe. In particular, the behavioural and emotional impacts must be measured and evaluated both on the actors and the people to whom they are addressed.

\section{Conclusion}

"Time is a concept developed to represent the variation of the world: the Universe is never frozen, the elements that compose it move, transform and evolve for the observer that is man. If we consider the Universe as a system as a whole, the observer finds that it has several states. These states, past, present and future, and their measurement, contribute to giving a concept of time [18]."

Can the virtual not also be considered as a non-frozen space? It is not touched, felt, seen or physically accessed. Wouldn't the past have a proportion not to make us leave the virtual world? Since only the moment seems to count because of the speed of access and obtaining what you want! Just as the future may just as easily be excluded from this virtual space since the projection does not seem to be ap- propriate in an environment where the click could be a temporal measure giving life to time following this measure. The time in the virtual whose definition must be considered has an impact on the behaviour of individuals behind the screen, especially because it is sometimes transposed into reality. Behavioural differentiations that net profiling reveals. How would Einstein, Kant, Galileo and others react and test their theory in this virtual universe?

I trust that new scientists will be able to redefine the notion of time in the virtual world, even by corroborating it with that considered in the real world. The impacts, particularly on human behaviour, will certainly be beyond what has been discovered to date, integrating my own research work. The evolution of virtual space and the elements integrating it in addition to the human, means favouring our lives are in such a mutation that it is possible to the notion of time in the virtual is still evolving.

The calendar is an organizational code in a spatial and temporal space. The Gregorian calendar prevails without questioning any reference point for our civilization over all other calendars. Time management also used in virtual space. The Gregorian calendar, which is based on physical data, applies to our three-dimensional space-time system. There is no time limit. According to our vision, education and culture transmitted from generation to generation, the minute, the day, the month, the year, ends to make the next period begin in a constant loop.

The Mayans have a spiritual vision that translates each endless event of action/reaction with an outcome that gives rise to a new process without being a succession of the previous one. This coincides with quantum physics, that is, there are different time scales in our universe in the same way that there are different overlapping space-time systems that can operate simultaneously.
Wouldn't the virtual be one that would force us to accept it and integrate it into our redefinition of time, which is perhaps one of the factors forcing us to change our behaviour in this new space?

\section{References}

1. Galileo is an Italian Mathematician, Geometer, Physicist and Astronomer of the $18^{\text {th }}$ century.

2. Limited relativity is the formal theory developed by Albert Einstein (theorist, physicist) in 1905 to draw all the physical consequences of Galilean relativity and the principle that the speed of light in vacuum has the same value in all Galilean referential (or inertial), which was implicitly stated in Maxwell's equations (but interpreted much differently until then, with Newton's "absolute space" and the ether). Galilean relativity states, in modern language, that any experience made in an inertial reference frame is carried out in a perfectly identical way in any other inertial reference frame. Having become the "principle of relativity", its statement will then be modified by Einstein to be extended to non-inertial referential: from "restricted", relativity will become "general", and will also deal with gravitation, which is not the case with restricted relativity.

3. Emmanuel Kant: Born on April 22, 1724 in Königsberg, capital of East Prussia, and died in the same city on February 12, 1804, is a German philosopher.

4. Touzeau N (2017) Behavioral cybercriminals differentiations between the real world and the virtual space. J Forensic Res 8(6): 401

5. Touzeau N (2018) Transposition of modus operandi from the real to the virtual using several signatures: Case of the drowned of the garonne serial crimes in France. J Forensic Sci \& Criminal 3(1): 1-2.

6. Touzeau N (2018) Avatarization another way to understand cyberbullyers behaviour in the real and the virtual world. Journal of Cognitive Neuropsychology 2(1): 1-2.

7. Touzeau N (2018) Transverse Zone: Explanation and definition in comparison with comfort zones. J Forensic Sci \& Criminal 9(1): 555753.

8. Touzeau N (2018) Virtual intelligence the ninth family of intelligences to be added to howard gardner's list. Crim Forensic Studies 1(1)

9. Touzeau N (2018) Some behavior differences between bullying and cyber bullying and impacts on adult victims. Crim Forensic Studies 1(2).

10. Touzeau N (2018) Can the definition of lying as known in real space be applied to lies perpetuated in the virtual, particularly with regard to the behavioural differentiations that virtual space promotes? Int J Forens Sci $3(2)$

11. Touzeau N (2018) Author of scientific books: "Netprofiling: analyse comportementale des cybercriminels", Amazon editor, Oct 2015 - "Net-profiling" Transition Editor.

12. Héraclite d'Éphèse, is a Greek philosopher from the end of the century BC.

13. Atomism is a philosophical current and a physical theory proposing a conception of a discontinuous universe, composed of matter and emptiness. According to atomists, the atoms in the universe are all of the same substance. They are unbreakable and differ from each other only in their shape, position and movement (today what we have called "atom" - which means "unbreakable" - is breakable - nuclear fission - but elementary particles are not in the current state of our knowledge). Atomists constitute reality with non- being, which thus has as much reality as being itself. In the $5^{\text {th }}$ century BC, Leucippus and his pupil Democritus of Abdère were considered to be the founders of atomism, a doctrine later adopted by Epicurus since the beginning of the $4^{\text {th }}$ century $\mathrm{BC}$, then by Lucretia ( $1^{\text {st }}$ century BC)2.

14. Augustine of Hippo or Saint Augustine, born in Thagaste, a municipality in the province of Africa, on 13 November 354, and died on 28 August 430 in Hippo, is a philosopher and theologian. 
15. Morvan SALEZ holds a doctorate in astrophysics and space technology. A former CNRS researcher, he received the Antoine d'Abbadie Prize from the French Academy of Sciences for the creation, with his team, of an instrument from the Herschel Space Observatory, launched by an Ariane rocket in 2009. He is interested in a wide range of research from fundamental physics to biology. He is also a scientific advisor, novelist, scriptwriter, singer-songwriter, activities to which he has been dedicated since 2012 .
16. Klein E. Director of the Laboratoire des Recherches sur les Sciences de la Matière (LARSIM) of the Commissariat à l'énergie atomique (CEA), Étienne Klein is also the author of numerous books, Saclay, France.

17.https://lesbrindherbes.org/2017/01/16/debat-apres-lesvieux-la-generation-y/

18. https://www.techno-science.net/definition/3651.html

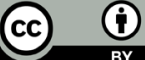

Creative Commons Attribution 4.0 International License

For possible submissions Click Here
Submit Article

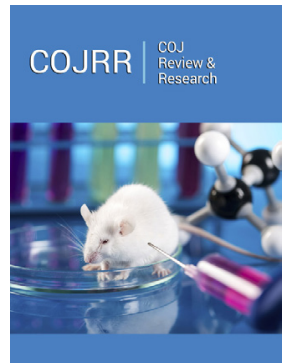

\section{COJ Reviews \& Research}

\section{Benefits of Publishing with us}

- High-level peer review and editorial services

- Freely accessible online immediately upon publication

- Authors retain the copyright to their work

- Licensing it under a Creative Commons license

- Visibility through different online platforms 